BMJ Open

Diabetes

Research

\& Care

\title{
Safety and efficacy of saxagliptin for glycemic control in non-critically ill hospitalized patients
}

\author{
Rajesh Garg, Brooke Schuman, Shelley Hurwitz, Cheyenne Metzger, \\ Shreya Bhandari
}

To cite: Garg R, Schuman B, Hurwitz $\mathrm{S}$, et al. Safety and efficacy of saxagliptin for glycemic control in noncritically ill hospitalized patients. BMJ Open Diabetes Research and Care 2017;5: e000394. doi:10.1136/ bmjdrc-2017-000394

Received 23 January 2017 Revised 2 March 2017 Accepted 9 March 2017

\section{ABSTRACT}

Objective: To evaluate whether saxagliptin is non-inferior to basal-bolus insulin therapy for glycemic control in patients with controlled type 2 diabetes mellitus (T2DM) admitted to hospital with non-critical illnesses.

Research design and methods: This was an open-label, randomized controlled clinical trial. Patients received either saxagliptin or basal-bolus insulin, both with correctional insulin doses. The main study outcome was the mean daily blood glucose (BG) after the first day of randomization.

Results: Of 66 patients completing the study, 33 (age $69 \pm 10$ years, $40 \%$ men) were randomized to saxagliptin and 33 (age $67 \pm 10$ years, $52 \%$ men) to basal-bolus insulin therapy. The mean daily BG was $149.8 \pm 22.0 \mathrm{mg} / \mathrm{dL}$ in the saxagliptin group and 146.9 $\pm 30.5 \mathrm{mg} / \mathrm{dL}$ in the insulin group $(\mathrm{p}=0.59)$. With an observed group difference of $2.9 \mathrm{mg} / \mathrm{dL}$ and an a priori margin of $20 \mathrm{mg} / \mathrm{dL}$, inferiority of saxagliptin was rejected in favor of non-inferiority $(p=0.007)$. There was no significant difference in the percentage of high or low BG values. The insulin group received a higher number of insulin injections $(2.3 \pm 1.7 /$ day vs $1.2 \pm 1.9$ / day; $p<0.001)$ as well as a higher daily insulin dose $(13.3 \pm 12.9$ units/day vs $2.4 \pm 3.3$ units/day; $p<0.001)$ than did the saxagliptin group. Continuous $B G$ monitoring showed that glycemic variability was lower in the saxagliptin group as compared to the insulin group. Patient satisfaction scores were similar in the two groups.

Conclusions: We conclude that saxagliptin use is non-inferior to basal-bolus insulin in non-critically ill hospitalized patients with T2DM controlled on 0-2 oral agents without insulin. Saxagliptin use may decrease glycemic variability in these patients.

Trial registration number: NCT02182895.
Division of Endocrinology, Diabetes, and Hypertension, Brigham and Women's Hospital, Harvard Medical School, Boston, Massachusetts, USA

Correspondence to Dr Rajesh Garg; rgarg@partners.org

\section{INTRODUCTION}

The relationship between inpatient hyperglycemia and poor clinical outcomes has been demonstrated in several observational studies. $^{1-5}$ Treatment of hyperglycemia is associated with decreased mortality and morbidity among hospitalized patients. ${ }^{6-8}$ On the basis of these data, good glycemic control in

\section{Significance of this study}

What is already known about this subject?

- Oral anti-diabetic agents are not currently recommended for treatment of diabetes in the hospital setting.

- Previous studies have shown sitagliptin use with basal insulin to be non-inferior to basal bolus insulin therapy.

\section{What are the new findings?}

- DPP-4 inhibitors can be used safely and effectively without insulin in a subgroup of hospitalized patients with well-controlled diabetes before admission.

- Use of DPP-4 inhibitors may decrease the variability in glucose levels among hospitalized patients.

- Use of DPP-4 inhibitors may reduce insulin use and increase comfort for patients.

How might these results change the focus of research or clinical practice?

- DPP-4 inhibitors, either alone or with basal insulin, should be allowed for inpatient glycemic control.

- Further research needs to investigate the glycemic control in hospitalized patients with mild diabetes without any treatment.

- Impact of non-insulin agent use on clinical outcomes in hospitalized patients needs further research.

hospitalized patients has been emphasized by many professional organizations. ${ }^{9} 10$ The current American Diabetes Association (ADA) guidelines recommend insulin as the preferred treatment for hospitalized patients. ${ }^{11}$ It is recommended that most critically ill patients should receive insulin infusion therapy and non-critically ill patients should receive basal-bolus insulin therapy in the hospital. Non-insulin hypoglycemic agents are not recommended due to multiple contraindications against many of these agents in acutely ill patients. For example, insulin secretagogues can cause hypoglycemia due to poor 
and unreliable nutritional intake in a hospitalized patient. Metformin and sodium glucose transporter (SGLT)-2 inhibitors are contraindicated in the presence of renal insufficiency or in patients at risk of developing renal insufficiency. Thiazolidinediones take a long time to act and are contraindicated in the presence of congestive heart failure or hepatic dysfunction. Moreover, many non-insulin agents including metformin and GLP-1 agonists can cause gastrointestinal side effects that are undesirable in an already sick hospitalized patient.

None of the above contraindications apply to dipeptidyl peptidase-4 (DPP-4) inhibitors. If effective, DPP-4 inhibitors may be preferable over insulin because of the low risk of hypoglycemia. Hypoglycemia is associated with increased mortality and morbidity among hospitalized patients, ${ }^{12-14}$ and ADA guidelines strongly recommend avoiding hypoglycemia. ${ }^{11}$ DPP-4 inhibitors may also reduce glycemic variability that is associated with poor clinical outcomes. ${ }^{15} 16$ Therefore, we conducted a study with the aim of testing the safety and efficacy of DPP-4 inhibitor, saxagliptin, in non-critically ill hospitalized patients with type 2 diabetes mellitus (T2DM).

\section{Study design and methods}

This was an open label randomized controlled clinical trial (ClinicalTrials.gov identifier NCT02182895) conducted at a single center. The Partners HealthCare Institutional Review Board approved the study protocol, and all participants provided a written informed consent. Patients older than age 18 years with T2DM and $\mathrm{HbA} 1 \mathrm{C} \leq 7.5 \%$ on a $\leq 1$ non-insulin hypoglycemic agent or $\mathrm{HbA} 1 \mathrm{C} \leq 7.0 \%$ on $\leq 2$ non-insulin hypoglycemic agents were enrolled into the study after admission to the hospital for a non-critical illness. HbAlc was measured at the time of admission (unless available within the past 3 months) in all patients with diabetes as a standard of care. Exclusion criteria included admission to the intensive care unit (ICU), a history of diabetic ketoacidosis or hyperosmolar state, insulin treatment before admission to hospital, unable to take oral food or medications, systemic steroid use, pregnancy or breastfeeding, a history of pancreatitis or active gallbladder disease, end-stage renal disease on dialysis, hypersensitivity to saxagliptin or another contraindication against saxagliptin, and inability to provide an informed consent.

Eligible participants were randomized by computergenerated numbers to one of the two groups: (1) DPP-4 inhibitor therapy: saxagliptin and (2) standard therapy: basal-bolus insulin regimen. The study statistician generated the randomization scheme using the web site randomization.com and kept it hidden. The investigators were unaware of the treatment assignment until the participant had signed the consent form and was determined to be eligible for the study.

The DPP-4 inhibitor therapy group received saxagliptin $5 \mathrm{mg}$ daily except for patients with eGFR $<50 \mathrm{~mL} /$ min or using strong CYP3A4/5 inhibitors (eg, atazanavir, clarithromycin, indinavir, itraconazole, nefazodone, nelfinavir, ritonavir, saquinavir, telithromycin) who received saxagliptin $2.5 \mathrm{mg}$ daily. Patients in the standard therapy group received basal-bolus insulin treatment at a starting dose of 0.5 units $/ \mathrm{kg} /$ day, given half as insulin glargine once daily and half as insulin aspart divided into three equal doses before meals. However, a lower insulin dose was allowed at the discretion of the treating team if oral intake was poor or unpredictable. The goal of therapy was to maintain a fasting blood glucose (BG) concentration between 70 and $140 \mathrm{mg} / \mathrm{dL}$ and all other BG values $<180 \mathrm{mg} / \mathrm{dL}$. The doses of insulin were adjusted daily by $10-20 \%$ to achieve these goals as per the standard practice.

In addition, both groups received the correctional sliding scale insulin therapy with insulin aspart before each meal and bedtime starting with 1 unit at BG $>150 \mathrm{mg} / \mathrm{dL}$ and increasing by 1 unit for each $50 \mathrm{mg}$ increment. Point of care BG levels were monitored before meals and at bedtime as per current standard practice. As a safety measure, two consecutive BG values $>200 \mathrm{mg} / \mathrm{dL}$ in the saxagliptin arm led to withdrawal from the study and a switch to the basal-bolus insulin regimen. Patients were also withdrawn from the study if transferred to the ICU, started on systemic glucocorticoids, or became unable to take oral meals.

The primary outcome of this study was the mean daily BG level obtained by point of care testing during study days 2-5. For those discharged before day 5, data were collected until the time of discharge. For those staying longer than 5 days, the study was stopped on day 6 and patients were switched to standard care with basal-bolus insulin therapy. Secondary outcomes included proportion of BG readings in $70-140 \mathrm{mg} / \mathrm{dL}$ range, average dose and number of insulin injections, incidence of hypoglycemia (BG $<70 \mathrm{mg} / \mathrm{dL}$ ), incidence of severe hypoglycemia ( $\mathrm{BG}<50 \mathrm{mg} / \mathrm{dL}$ ), incidence of hyperglycemia (BG $>200 \mathrm{mg} / \mathrm{dL}$ ), treatment failure with DPP-4 inhibitor, and length of hospital stay.

All patients enrolled in the study were asked if they would agree to a continuous glucose monitoring (CGM) insertion. A subset of patients agreed and underwent CGM (iPRO2, Medtronic) to obtain data for glycemic variability. Glucose SD, mean amplitude of glycemic excursions (MAGE) and continuous overlapping net glycemic action (CONGA) were derived from the CGM data using software developed by Hill et al. ${ }^{17} 18$

A well-validated inpatient diabetes treatment satisfaction questionnaire (DTSQ-IP), items 1-16, was administered before the time of discharge. ${ }^{19}$ This questionnaire had 16 items that were scored on a scale of $0-6$. For all the items except for items 2 and 3, ' 0 ' indicates lowest satisfaction and ' 6 ' indicates highest satisfaction. For items 2 and 3, ' 0 ' indicates highest satisfaction and ' 6 ' indicates lowest satisfaction.

\section{Statistical analysis}

With the mean daily BG as the main outcome, a sample size of 33 randomized to each treatment arm was 
determined to achieve at least $80 \%$ power to test the null hypothesis of saxagliptin inferiority relative to basal-bolus insulin, with $20 \mathrm{mg} / \mathrm{dL}$ as the a priori non-inferiority margin, and a within-group SD of $31 \mathrm{mg} / \mathrm{dL}^{20}$ The number enrolled was 74 because of dropouts and missing data for some. Along with the noninferiority hypothesis test for the mean daily BG, the upper limit of a one-sided 95\% CI around the observed difference between arms was presented. The mean daily BG level during hospital included days 2-5. Day of enrollment into the study was defined as hospital day 1 . All continuous data were summarized as mean \pm SD and categorical data as number with percent. The WilcoxonMann-Whitney test was used to compare continuous variables, and the $\chi^{2}$ test was used to compare categorical variables. Data were analyzed using software SAS V.9.4.

\section{RESULTS}

Of 74 patients who signed an informed consent form, 2 withdrew consent before the first dose of the study drug and 6 were discharged within 24 hours of enrollment into the study (figure 1). Complete data were available for 33 patients in the saxagliptin group and 33 patients in the basal-bolus insulin group.

Baseline patient characteristics are shown in table 1 . All patients had well-controlled diabetes on 0-2 oral agents before admission to hospital and had relatively mild hyperglycemia at the time of randomization. The majority of patients were admitted under a surgical service. There were no differences in any of the baseline variables between the two groups. The main study outcomes are shown in table 2. The primary outcome was not different between the two groups, and the noninferiority criteria for saxagliptin were satisfied. With an observed group difference of $2.9 \mathrm{mg} / \mathrm{dL}$ and an a priori non-inferiority margin of $20 \mathrm{mg} / \mathrm{dL}$, the null hypothesis

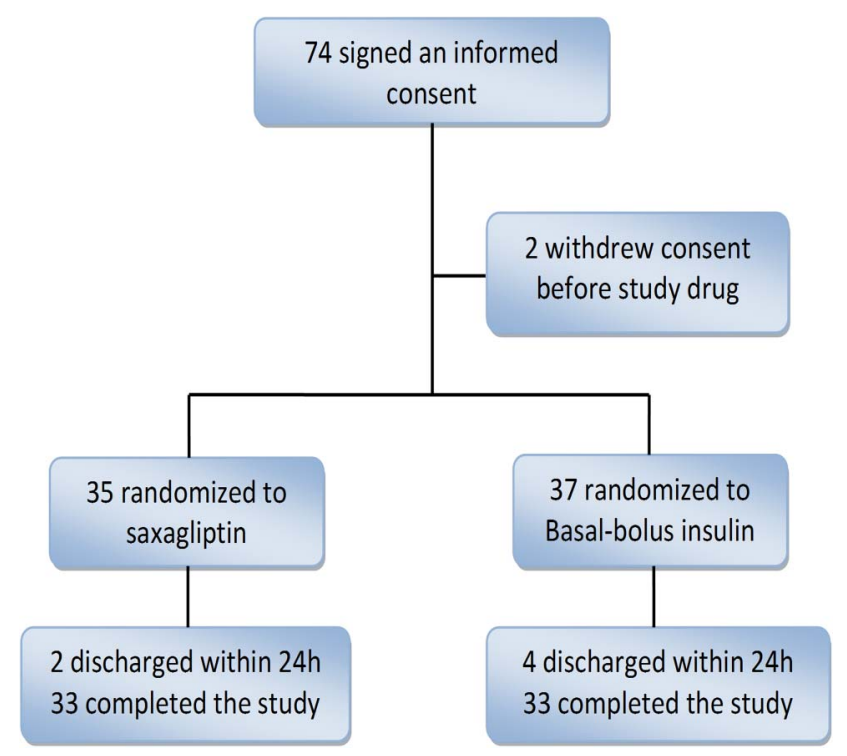

Figure 1 Patient flow through the study. of inferiority was rejected in favor of the alternative hypothesis of non-inferiority $(\mathrm{p}=0.007)$. Further, the upper limit of a one-sided 95\% CI around the difference between group means was $14.2 \mathrm{mg} / \mathrm{dL}$, well within the non-inferiority margin.

Other glycemic control indices were also similar between the two groups. However, insulin use, dose as well as the number of insulin injections, was significantly higher in the basal-bolus insulin group as compared to the saxagliptin group. Seven patients in the saxagliptin group and eight patients in the insulin group had BG $>180 \mathrm{mg} / \mathrm{dL}$ at the time of randomization. Among these patients, the mean daily BG values (days 2-5) were 163.4 \pm 19.0 in the saxagliptin group and $161.7 \pm 38.0$ in the insulin group $(\mathrm{p}=0.86)$.

The groups had similar BG levels on day 1. However, over the course of the study, the saxagliptin group showed a downward trend in BG levels while the control group showed a decrease on day 2 and then stable BG values despite an increasing dose of insulin (figure 2). One patient in the saxagliptin group was withdrawn from the study and switched to basal bolus insulin due to two consecutive $\mathrm{BG}$ values $>200 \mathrm{mg} / \mathrm{dL}$. For this patient, data before switching to insulin were included in analysis. One patient in each group had one episode of BG $<70 \mathrm{mg} / \mathrm{dL}$. No patient in either group developed severe hypoglycemia.

Continuous BG monitoring data were available in 36 patients, 20 in the saxagliptin group and 16 in the control group. Glucose SD and MAGE were lower in the saxagliptin group as compared to the insulin group (table 3). CONGA values were similar in the two groups.

There was no difference in DTSQ-IP scores between the groups. The saxagliptin and control groups rated their diabetes inpatient treatment between $80 \%$ and $90 \%$ of the satisfaction score with no significant difference between the average scores. Similarly, there was no significant difference in inpatient dissatisfaction (blood sugars being unacceptably high or low) between the two groups. No patient needed additional surgery or was transferred to the ICU while enrolled into the study. Five patients in the saxagliptin group had known heart disease, and they were watched closely for heart failure. No new-onset heart failure or worsening of heart failure was observed in these patients.

\section{DISCUSSION}

This study shows that saxagliptin is non-inferior to basalbolus insulin for glycemic control in non-critically ill hospitalized patients with well-controlled diabetes before admission. There is no risk of hypoglycemia associated with saxagliptin use. Moreover, glycemic variability is lower with saxagliptin therapy than with basal-bolus insulin therapy. The study is important because current clinical practice guidelines recommend insulin for management of all hyperglycemia in hospitalized patients. ${ }^{11}$ We estimate that a substantial number of hospitalized 
Table 1 Baseline characteristics

\begin{tabular}{|c|c|c|c|}
\hline & $\begin{array}{l}\text { Saxagliptin group } \\
\mathrm{N}=33\end{array}$ & $\begin{array}{l}\text { Basal-bolus insulin group } \\
\mathrm{N}=33\end{array}$ & p Value \\
\hline Age, years & $69 \pm 10$ & $67 \pm 10$ & 0.16 \\
\hline Gender, N (\%) & & & 0.32 \\
\hline Male & $13(40)$ & $17(52)$ & \\
\hline Female & $20(60)$ & $16(48)$ & \\
\hline Race, N (\%) & & & 0.23 \\
\hline White & $26(79)$ & $30(91)$ & \\
\hline Other & $7(21)$ & $3(9)$ & \\
\hline Admitting service, $\mathrm{N}(\%)$ & & & 0.16 \\
\hline Medicine & $6(18)$ & $11(33)$ & \\
\hline Surgery & $27(82)$ & $22(67)$ & \\
\hline Duration of diabetes, years & $7.5 \pm 8.5$ & $7.5 \pm 5.8$ & 0.64 \\
\hline Mean A1c, \% & $6.6 \pm 0.5$ & $6.5 \pm 0.5$ & 0.69 \\
\hline Preadmission diabetes medications, N (\%) & & & 0.06 \\
\hline None & $8(24)$ & $5(15)$ & \\
\hline Metformin & $22(67)$ & $18(55)$ & \\
\hline SU & $2(6)$ & $10(30)$ & \\
\hline Other & $1(3)$ & 0 & \\
\hline Weight, kg & $97.7 \pm 32.9$ & $92.4 \pm 21.2$ & 0.54 \\
\hline BMI & $34.5 \pm 12.2$ & $32.6 \pm 6.5$ & 0.91 \\
\hline Admission blood glucose, $\mathrm{mg} / \mathrm{dL}$ & $156.1 \pm 78.9$ & $152.8 \pm 52.5$ & 0.52 \\
\hline Prerandomization blood glucose, $\mathrm{mg} / \mathrm{dL}$ & $154.6 \pm 37.3$ & $154.8 \pm 54.6$ & 0.97 \\
\hline Serum creatinine & $1.02 \pm 0.34$ & $1.10 \pm 0.35$ & 0.26 \\
\hline Presence of infection, N (\%) & $4(12)$ & $8(24)$ & 0.20 \\
\hline
\end{tabular}

BMI, body mass index.

\section{Table 2 Outcome variables}

\begin{tabular}{|c|c|c|c|}
\hline & $\begin{array}{l}\text { Saxagliptin group } \\
\mathrm{N}=33\end{array}$ & $\begin{array}{l}\text { Basal-bolus insulin group } \\
\mathrm{N}=33\end{array}$ & p Value* \\
\hline Mean $B G$ on day $1 \mathrm{mg} / \mathrm{dL}$ & $154.8 \pm 28.2$ & $156.0 \pm 32.1$ & 1.0 \\
\hline Mean daily $B G$ day $2-5 \mathrm{mg} / \mathrm{dL} \dagger$ & $149.8 \pm 22.0$ & $146.9 \pm 30.5$ & 0.59 \\
\hline BGs in $70-140, N(\%)$ & $102(42)$ & $105(37)$ & 0.16 \\
\hline Number of patient days with at least one $B G>200, N(\%)$ & $14(15)$ & $22(19)$ & 0.27 \\
\hline BGs $>200, N(\%)$ & $17(6)$ & $31(10)$ & 0.16 \\
\hline Number of patient days with at least one $B G<70$ & 1 & 1 & \\
\hline BGs $<70(\%)$ & 0.4 & 0.3 & NS \\
\hline Number of patient days with at least one $B G<50$ & 0 & 0 & \\
\hline BGs $<50(\%)$ & 0 & 0 & \\
\hline LOS, days & $8.0 \pm 14.6$ & $6.4 \pm 5.4$ & 0.87 \\
\hline \multicolumn{4}{|l|}{ Mean daily insulin dose, units } \\
\hline Total & $2.4 \pm 3.3$ & $13.3 \pm 12.9$ & $<0.001$ \\
\hline Basal & 0 & $6.1 \pm 7.2$ & \\
\hline Bolus & $2.4 \pm 3.3$ & $7.4 \pm 7.1$ & $<0.001$ \\
\hline Mean number of injections per day & $1.2 \pm 1.9$ & $2.3 \pm 1.7$ & $<0.001$ \\
\hline DTSQ IP composite score (range $0-84$ ) & $70 \pm 12$ & $75 \pm 11$ & 0.19 \\
\hline DTSQ IP Q2+Q3 score (range 0-12) & $3.9 \pm 3.7$ & $2.5 \pm 3.0$ & 0.12 \\
\hline
\end{tabular}

${ }^{*}$ Tests of superiority include the Wilcoxon rank sum test and $\chi^{2}$ test.

†Main study outcome for the non-inferiority test. With group difference of $2.9 \mathrm{mg} / \mathrm{dL}$ and a priori margin of $20 \mathrm{mg} / \mathrm{dL}$, we reject inferiority of saxagliptin in favor of non-inferiority $(p=0.007)$.

$B G$, blood glucose.

patients with diabetes may be eligible for treatment with non-insulin agents. Avoiding insulin in these patients can make the inpatient diabetes management much simpler for the nursing staff and may also alleviate anxiety for the patients. The frequency of BG testing may be also be decreased in these patients. Overall, this may save nursing time and translate into lower hospitalization costs while improving patient comfort. 
Mean Blood Glucose During Study
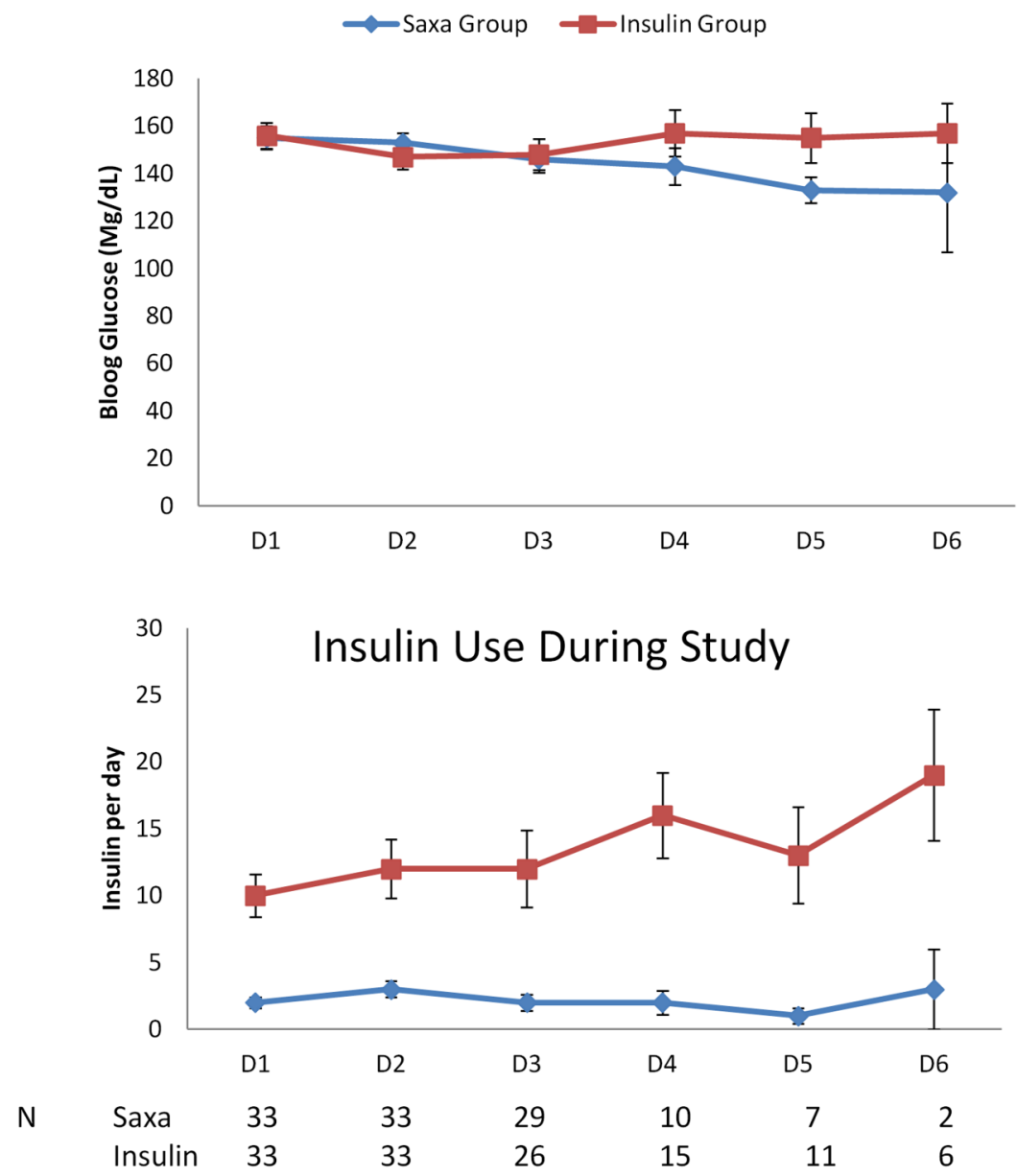

Figure 2 Mean blood glucose levels and insulin use during the course of the study.

Table 3 Indices of glycemic variability

\begin{tabular}{llll}
\hline & $\begin{array}{l}\text { Saxagliptin } \\
\text { group } \\
\mathbf{N}=\mathbf{2 0}\end{array}$ & $\begin{array}{l}\text { Basal-Bolus } \\
\text { insulin group } \\
\mathbf{N}=16\end{array}$ & p Value \\
\hline Glucose SD & $1.13 \pm 0.47$ & $1.61 \pm 0.73$ & 0.03 \\
MAGE & $2.72 \pm 1.60$ & $3.93 \pm 2.03$ & 0.05 \\
CONGA & $7.38 \pm 1.46$ & $7.56 \pm 1.58$ & 0.86 \\
\hline
\end{tabular}

CONGA, continuous overlapping net glycemic action; MAGE, mean amplitude of glycemic excursions.

A previous study by Umpierrez $e t a l^{20}$ showed sitagliptin use to be non-inferior to basal-bolus insulin in hospitalized patients. Their study enrolled patients irrespective of their baseline HbAlc or previous insulin treatment. In that study, patients with baseline BG $>180 \mathrm{mg} / \mathrm{dL}$ had higher mean daily BG levels during hospital stay when treated with sitagliptin alone as compared to treatment with insulin. Therefore, a subset of patients remained poorly controlled when treated with DPP-4 inhibitor alone irrespective of their baseline diabetes control. In a recent, multicenter, randomized controlled trial, the same group of investigators have shown that sitagliptin along with basal insulin is able to achieve glycemic control that is non-inferior to the basal-bolus insulin therapy. ${ }^{21}$ Thus, even in patients with relatively high BG levels at baseline, DPP-4 inhibitor use may obviate the need for nutritional insulin. We restricted our patient population to participants with milder hyperglycemia, who were most likely to respond to DPP-4 inhibitor therapy alone, using clinical criteria often used in the outpatient setting. Moreover, it has been shown that glycemic control prior to treating hyperglycemia in hospitalized patients can predict response to therapy. ${ }^{22}$ Only one patient was labeled as a non-responder in the saxagliptin group, thus demonstrating that this strategy was highly successful. Our study complements data generated by Umpierrez and colleagues on the safety and efficacy of DPP-4 inhibitors in the hospital setting and adds data on the glycemic variability. Our results are also consistent with the recently suggested algorithm for the use of DPP-4 inhibitors in patients with moderate hyperglycemia at admission. ${ }^{23}$

Patients in the basal-bolus insulin group showed no change in BG levels over the study period despite increasing insulin doses. This may be due to lower than 
recommended starting doses of insulin and a lag in escalating the insulin doses as the patients' oral intake improved. In this study, insulin doses were advised by the research team according to protocol but often cut back by the primary teams due to fear of hypoglycemia. Practically, fear of hypoglycemia (on the part of physicians) is one of the common reasons for insulin underdosing and relevant to our study because DPP-4 inhibitors do not cause hypoglycemia. Moreover, it is not uncommon for physicians to be cautious while adjusting insulin doses in the inpatient setting because oral intake is often unpredictable. Owing to the high risk of hypoglycemia with insulin, an increase in insulin doses is often based on the previous day's high BG numbers. This is a reasonable approach, even though it leads to overall high BG levels during the hospital stay. However, no dose adjustment is needed if DPP-4 inhibitor is used instead on insulin. Moreover, lower glycemic variability may be an additional advantage of using these agents in the inpatient setting as previous studies have demonstrated an association between higher glycemic variability and poor clinical outcomes irrespective of mean BG levels in hospitalized patients. ${ }^{15} 16$ The trend of lower BG levels during the course of study in our saxagliptin group remains unexplained; it may be due to the accumulating effect as the subsequent doses of saxgliptin were administered after day 1 .

One limitation of our study is the inability to evaluate clinical outcomes and to compare hospital complications between the two groups. Length of hospital stay was not different between the saxagliptin group and the basal-bolus insulin group. There has been some discussion whether the benefits of good glycemic control in acutely ill patients are the result of lower BG levels or a direct effect of insulin. ${ }^{24}$ If insulin has a direct effect independent of glucose levels, DPP-4 inhibitor therapy may not be able to decrease the risk of complications. A large, prospective, randomized, multicenter clinical trial would be necessary to investigate this hypothesis. Another limitation of our study is the lack of a placebo group. After completion of the study, we noticed that many of the enrolled participants had acceptable BG levels $(<180 \mathrm{mg} / \mathrm{dL})$ at the time of randomization. It is possible that their BG levels would have remained in acceptable range without any treatment. However, all these patients had known T2DM and as per current standards of clinical care, all of them should have received basal-bolus insulin therapy as inpatients. Nevertheless, physicians did not give insulin doses proposed by the study team. Insulin underdosing is one of the practical problems of inpatient diabetes management and a limitation of our study. We think future studies should include a placebo group to evaluate whether patients with baseline characteristics similar to those enrolled in this study may be left alone without any anti-diabetic treatment. It would also be worth comparing saxagliptin alone with a basal plus approach, used in the sitaglipitin studies. Another limitation of our study is the small sample size and unequal distribution of surgical and medical patients, making it hard to perform subgroup analyses, because the majority of our patients were admitted under surgical specialties and the results are not necessarily applicable to medical patients.

In conclusion, this study demonstrates that saxagliptin use is safe and effective when compared with basal-bolus insulin in a subgroup of non-critically ill hospitalized patients with well-controlled T2DM on 0-2 oral agents without insulin use before admission. Saxagliptin use may decrease glycemic variability in these patients. Thus, DPP-4 inhibitors may be an alternative to insulin use in a subgroup of hospitalized patients with diabetes. We propose that non-critically ill hospitalized patients with HbA1c $<7 \%$ on a $\leq 1$ oral anti-diabetic agent or HbAlc $<7.5 \%$ on a $\leq 2$ oral anti-diabetic agent should be treated with a DPP-4 inhibitor as a first step. Fasting BG should be monitored, and basal insulin should be added if BG $>140 \mathrm{mg} / \mathrm{dL}$. Multiple daily insulin injections are unnecessary in the majority of these patients.

Contributors RG designed the study, obtained funding, led the program, supervised the data collection, analyzed the data, and wrote the manuscript; $\mathrm{BS}, \mathrm{CM}$, and SB helped in conducting the study, collected the data, and reviewed the manuscript; $\mathrm{SH}$ conducted all statistical analysis and collaborated on writing the manuscript.

Funding This work was supported by AstraZeneca Company through an investigator-initiated research grant.

Competing interests $R G$ received research support from AstraZeneca for this study. Other authors have no conflict of interest.

Patient consent Obtained.

Ethics approval Partners Institutional Review Board

Provenance and peer review Not commissioned; externally peer reviewed.

Data sharing statement No additional data are available.

Open Access This is an Open Access article distributed in accordance with the Creative Commons Attribution Non Commercial (CC BY-NC 4.0) license, which permits others to distribute, remix, adapt, build upon this work noncommercially, and license their derivative works on different terms, provided the original work is properly cited and the use is non-commercial. See: http:// creativecommons.org/licenses/by-nc/4.0/

\section{REFERENCES}

1. Ishihara M, Kojima S, Sakamoto $\mathrm{T}$, et al. Acute hyperglycemia is associated with adverse outcome after acute myocardial infarction in the coronary intervention era. Am Heart J 2005;150:814-20.

2. Garg R, Bhutani H, Alyea E, et al. Hyperglycemia and length of stay in patients hospitalized for bone marrow transplantation. Diabetes Care 2007;30:993-4.

3. Umpierrez GE, Isaacs SD, Bazargan N, et al. Hyperglycemia: an independent marker of in-hospital mortality in patients with undiagnosed diabetes. J Clin Endocrinol Metab 2002;87:978-82.

4. Williams LS, Rotich J, Qi R, et al. Effects of admission hyperglycemia on mortality and costs in acute ischemic stroke. Neurology 2002;59:67-71.

5. Ascione R, Rogers CA, Rajakaruna C, et al. Inadequate blood glucose control is associated with in-hospital mortality and morbidity in diabetic and nondiabetic patients undergoing cardiac surgery. Circulation 2008;118:113-23.

6. Krinsley JS. Translating evidence into practice in managing inpatient hyperglycemia. J Hosp Med 2007;2(Suppl 1):13-19.

7. Umpierrez GE, Smiley D, Jacobs S, et al. Randomized study of basal-bolus insulin therapy in the inpatient management of patients 
with type 2 diabetes undergoing general surgery (RABBIT 2 surgery). Diabetes Care 2011;34:256-61.

8. Murad MH, Coburn JA, Coto-Yglesias F, et al. Glycemic control in non-critically ill hospitalized patients: a systematic review and meta-analysis. J Clin Endocrinol Metab 2012;97:49-58.

9. Moghissi ES, Korytkowski MT, DiNardo M, et al. American Association of Clinical Endocrinologists and American Diabetes Association consensus statement on inpatient glycemic control. Diabetes care 2009;32:1119-31.

10. Umpierrez GE, Hellman R, Korytkowski MT, et al. Management of hyperglycemia in hospitalized patients in non-critical care setting: an endocrine society clinical practice guideline. J Clin Endocrinol Metab 2012;97:16-38.

11. American Diabetes Association. 13. Diabetes Care in the Hospital. Diabetes Care 2016;39:S99-S104.

12. D'Ancona G, Bertuzzi F, Sacchi L, et al. latrogenic hypoglycemia secondary to tight glucose control is an independent determinant for mortality and cardiac morbidity. Eur J Cardiothorac Surg 2011;40:360-6.

13. Garg R, Hurwitz S, Turchin A, et al. Hypoglycemia, with or without insulin therapy, is associated with increased mortality among hospitalized patients. Diabetes Care 2013;36:1107-10.

14. Turchin A, Matheny ME, Shubina M, et al. Hypoglycemia and clinica outcomes in patients with diabetes hospitalized in the general ward. Diabetes Care 2009;32:1153-7.

15. Farrokhi $\mathrm{F}$, Chandra $\mathrm{P}$, Smiley $\mathrm{D}$, et al. Glucose variability is an independent predictor of mortality in hospitalized patients treated with total parenteral nutrition. Endocr Pract 2014;20:41-5.

16. Mendez CE, Mok KT, Ata A, et al. Increased glycemic variability is independently associated with length of stay and mortality in noncritically ill hospitalized patients. Diabetes Care 2013;36:4091-7.

17. Hill NR, Oliver NS, Choudhary $\mathrm{P}$, et al. Normal reference range for mean tissue glucose and glycemic variability derived from continuous glucose monitoring for subjects without diabetes in different ethnic groups. Diabetes Technol Ther 2011;13:921-8.

18. https://www.phc.ox.ac.uk/research/technology-outputs/easygv [article online].

19. Sampson MJ, Singh H, Dhatariya KK, et al. Psychometric validation and use of a novel diabetes in-patient treatment satisfaction questionnaire. Diabet Med 2009;26:729-35.

20. Umpierrez GE, Gianchandani R, Smiley D, et al. Safety and efficacy of sitagliptin therapy for the inpatient management of general medicine and surgery patients with type 2 diabetes: a pilot, randomized, controlled study. Diabetes Care 2013;36:3430-5.

21. Pasquel FJ, Gianchandani R, Rubin DJ, et al. Efficacy of sitagliptin for the hospital management of general medicine and surgery patients with type 2 diabetes (Sita-Hospital): a multicentre, prospective, open-label, non-inferiority randomised trial. Lancet Diabetes Endocrinol 2017;5:125-33.

22. Pasquel FJ, Gomez-Huelgas R, Anzola I, et al. Predictive value of admission hemoglobin A1c on inpatient glycemic control and response to insulin therapy in medicine and surgery patients with type 2 diabetes. Diabetes Care 2015;38:e202-203.

23. Nauck MA, Meier JJ. Sitagliptin plus basal insulin: simplifying in-hospital diabetes treatment? Lancet Diabetes Endocrinol 2017;5:83-5.

24. Garg R, Chaudhuri A, Munschauer F, et al. Hyperglycemia, insulin, and acute ischemic stroke: a mechanistic justification for a trial of insulin infusion therapy. Stroke 2006;37:267-73. 